Proceedings of the International Symposium on Physics of Materials (ISPMA 14), September 10-15, 2017, Prague

\title{
On changes of Monoclinic Twinning by Passage of Type II Twin Boundary in Ni-Mn-Ga 10M Martensite
}

\author{
V. KOPECKÝ* AND O. HECZKO
}

Institute of Physics, Czech Academy of Sciences, Na Slovance 2, 18221 Praha 8, Czech Republic

High mobility of a/c twin boundaries in $10 \mathrm{M}$ martensite of $\mathrm{Ni}-\mathrm{Mn}-\mathrm{Ga}$ is a unique phenomenon which can be used in various applications, e.g. actuation. The reason for the phenomenon may be the complex twinning system. This work is the first study of the interaction between monoclinic twinning and type II single twin boundary. We show at the level of optical microscopy that monoclinic twins crossing type II twin boundary are compatible enough to occur no or minor changes after type II twin boundary passage.

DOI: 10.12693/APhysPolA.134.867

PACS/topics: $61.72 . \mathrm{Mm}, 81.30 . \mathrm{Kf}, 81.40 . \mathrm{Jj}$

\section{Introduction}

Magnetic shape memory alloys exhibit several unique effects $[1,2]$, e.g. the material exhibits large reversible pseudoplastic deformation of few percent induced by moderate magnetic field called magnetically induced reorientation (MIR) [3, 4]. This phenomenon can be used for a plethora of applications, however, actuation is the most promising one $[5,6]$.

Magnetic shape memory effect is underlined by the existence of diffusionless martensitic transformation [1]. Martensitic transformation is a reversible displacive transition with stable chemical composition and inherited ordering [7]. Parent high-symmetry phase called austenite transforms to the lower-symmetry low-temperature phase called martensite by nucleation and growth of martensite nuclei forming a phase interface $[8,9]$. Due to different symmetry and lattice parameters, the martensitic and austenitic phases are not directly compatible [10]. The incompatibility at the phase interface is decreased by twinning of martensitic phase. A specific twinning is important for compatibility and thus reversibility of martensitic transformation.

In $10 \mathrm{M}$ modulated martensite of $\mathrm{Ni}-\mathrm{Mn}-\mathrm{Ga}$ three levels of twinning structure were identified by X-ray diffraction $[11,12]$ and theoretically described in $[13,14]$. The macroscopic level is occupied a/c twinning boundaries which are highly mobile and enable magnetic shape memory effect by reorientation of the crystal structure. The a/c boundaries can exist in two types, type I and II, that differ in their mobility [15]. Mesoscopic monoclinic (also called modulation) twinning flips the modulation and monoclinicity. Monoclinic twin boundary in thin foil studied by TEM shows curved and step-like interface [16] but in the bulk it seems to be straight $[17,18]$ forming various lamellar patterns. The monoclinic twinning under certain conditions can compatibly intersect a/c twin boundary $[11,19]$. Due to this fact, we now know that the monoclinic twins could be in past mis-

*corresponding author; e-mail: kopeckyv@fzu.cz interpreted and marked as 180 deg. magnetic domains, e.g. in Ref. [20]. Both, monoclinic and a/c twinning, can be observed by optical microscopy which is shown in the present work. Finally, at the microscopic level an a/b twinning can be found which is beyond the resolution of optical microscopy, and which was recently experimentally observed by SEM [21].

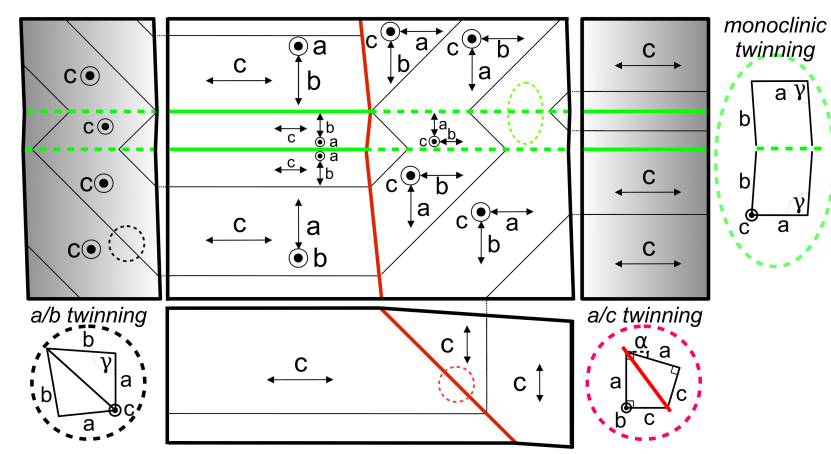

Fig. 1. Sketch of the possible twinning complex in 10M martensite according to the model [11]. The schematic drawings of all three levels of twinning are shown and their corresponding places are circled in the sketch. All angles and lattice parameters differences are exaggerated to be illustrative, as well as the a/b laminate width which is in reality on the sub-micron scale [21].

In this work we use the model proposed in Ref. [11] and further developed by Seiner et al. [13]. The model tells us that e.g. type II twin boundary lying in approximately (10 1 10) plane is compatible with the monoclinic twin in (010) plane. The model very well corresponds to our observations and helps to identify types of twin boundaries. In addition, we provide a sketch of the twinned complex which is shown in Fig. 1. The sketch is based on the model and depicted according to our observation.

This paper brings the first experimental observation of mutual dynamical relation between monoclinic twinning and type II single twin boundary. We investigate the changes in monoclinic twin microstructure after the passage of single type II boundary induced by mechanical stress. However, the change must be large enough to be detected by the optical microscope. 


\section{Methods}

In our experiment we used $\mathrm{Ni}_{50} \mathrm{Mn}_{28.7} \mathrm{Ga}_{21.3}$ single crystal sample produced by Adaptamat Ltd. The sample had the parallelogram shape with size about $20 \times 2 \times 1 \mathrm{~mm}$ and was cut approximately along $\{100\}$ planes of the parent phase. Transformation temperature from ten layered martensite $(10 \mathrm{M})$ to austenite was at $328 \mathrm{~K}$. The observation was performed at room temperature at which the martensite was stable. The lattice parameters of martensite in the monoclinic approximation were $a=0.5843 \mathrm{~nm}$, $b=0.5974 \mathrm{~nm}, c=0.5589 \mathrm{~nm}, \alpha=\beta=90, \gamma=90.34$ degrees at room temperature.

The sample was put into nitric acid for few seconds to remove any surface stresses. Unfortunately the acid also enhanced crystal defects making the surface uneven. Moreover, the mechanical testing resulted in unavoidable scratches. However, it did not affect the twins recognition and information brought by the study. The macroscopic single a/c twin boundary was formed by mechanical means and moved by mechanical stress applied along the long side of the sample, or approximately along monoclinic twin boundary.

Micrographs were taken by optical microscope Zeiss using Nomarski contrast (C-DIC) which visualizes the different tilt of surface planes in different colours. In our case the tilt originates from various twinning. The surface angle between two variants connected with a/c twin boundary is given approximately by $\alpha=90-$ $2 \arctan (c / a)$, i.e. about 3 deg. The surface relief due to monoclinic twinning is twice the monoclinic distortion, i.e. about $0.7 \mathrm{deg}$. To visualize twinning that does not make surface relief we used linearly polarized light. Observed contrast arises due to optical activity of differently oriented twin variants. However, due to the small difference between variants the contrast is quite weak.

Polarization contrast pictures are shown in shades of blue colour and Nomarski contrast in various colours. Additionally, pictures of the whole sample presented in Fig. 2 was merged from several micrographs in order to increase the final resolution of the pictures and to bring the whole perspective of the sample into the discussion.

\section{Results and discussion}

In contrast to previous papers, we studied the twinning on both sides of the sample. This way we make certain that we do not observe just surface effect and also evaluate properly the extent of the twinning. Figure 2 consists of two parts (A and B) showing both sides of the sample. Each part contains three different large micrographs: the upper picture (a) is taken using Nomarski contrast with single a/c twin boundary in its initial position; the second picture (b) with polarization contrast showing the boundary in the final position after mechanical stress; and the lowest part (c) is post-processed by application of threshold filter to enhance polarization contrast.

On both sides of the sample (A and B) a single a/c twin boundary oriented vertically is clearly visible on the

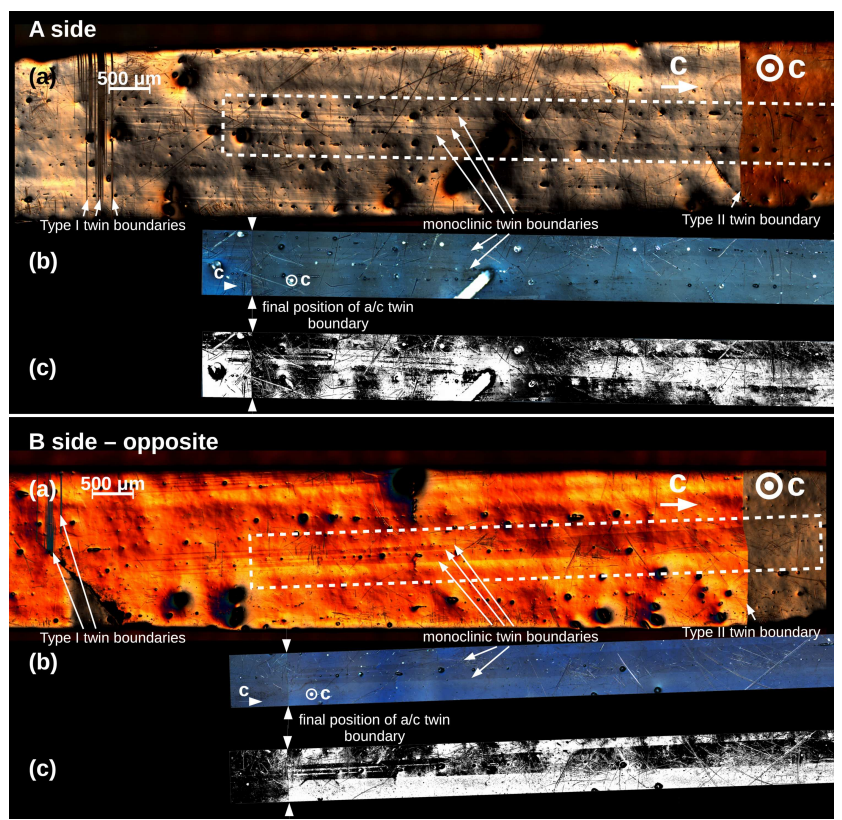

Fig. 2. Optical micrographs of both sides (A and B side) of the sample before (a) and after (b) passage of type II a/c twin boundary induced by tensile stress. The micrographs were taken using Nomarski (or C-DIC) contrast (a) and polarization contrast (b). Monoclinic twinning is emphasized by the dashed rectangle which also marks an area of polarization contrast picture. In order to increase the contrast of the (b) picture a threshold filter was applied (c). On the right side of (a) is type II twin boundary (vertical) and it is crossed by two monoclinic twin boundaries (horizontal). The crossing creates so-called zigzag pattern at the type II twin boundary. In part (b), the same type II twin boundary is moved by tensile stress into the position marked by two vertical arrows. The side B micrographs are presented as taken and no flipping was applied, thus the monoclinic traces are mirrored. In order to increase the resolution of the pictures they are composed of many smaller micrographs. The scale is the same for all pictures.

right side of the sample (Fig. 2a). The a/c twin boundary is deflected in two points at which two horizontal twin boundaries intersect it. The horizontal twin boundaries are monoclinic twins and the vertical one is type II twin boundary [13]. This interpretation is schematically depicted in Fig. 1.

From the Fig. 2 is clear that both types of twin boundaries are macroscopic $2 \mathrm{D}$ object extending through whole sample volume. The twin boundaries on both sides exhibit only minor differences, therefore, in further text we will discuss only side A as both sides are almost identical.

The monoclinic twinning produces a surface relief on the left side from the type II twin boundary where $c$ axis is oriented horizontally and in- plane (Fig. 2a). The surface tilt (about $0.7 \mathrm{deg}$ ) is visible as single horizontal stripe of a different shade than the background. Towards the left the single monoclinic twin branches forming needle-like twins and then disappearing. The termination is due to type I twin boundary located at the left 
side of the sample as the monoclinic twins in this orientation are incompatible and cannot cross type I twin boundary [13].

On the right side of the Fig. 2a the monoclinic twin boundaries cross type II twin boundary at two points. The trace of type II twin boundary changes direction at the intersections and forms so-called zigzag pattern [11]. The monoclinic twin continues after crossing the type II twin boundary, however, it is not visible by Nomarski contrast as there is no surface tilt. The proof of twin continuity is demonstrated on the polarization contrast picture which covers the area inside the dashed rectangle (Fig. 2b) The experimental proof of monoclinic twin continuity crossing type II twin boundary was presented by Heczko et al. [21] and it is drawn in Fig.1.

This initial state was changed by application of small tensile stress (about 0.5 MPa). The only difference in the polarization contrast picture (Fig. 2b) is that the same type II twin boundary is moved to the left by applied tensile stress and placed as marked by arrows. Presenting the polarization contrast picture it is clear that the monoclinic twin continues through the position of the previous intersection. Defects of the sample can be used as markers when comparing the pictures. From the comparison it can be seen that the defects are more distant in the horizontal axis and thus the sample is longer in picture (b) than in picture (a). It is caused by reorientation of the microstructure which takes place by the passage of a mobile twin boundary, i.e. extension of $6 \%$, induced by tensile stress. To increase the weak contrast of polarization pictures and enhance visibility we post-processed the picture by applying a threshold filter (Fig. 2c).
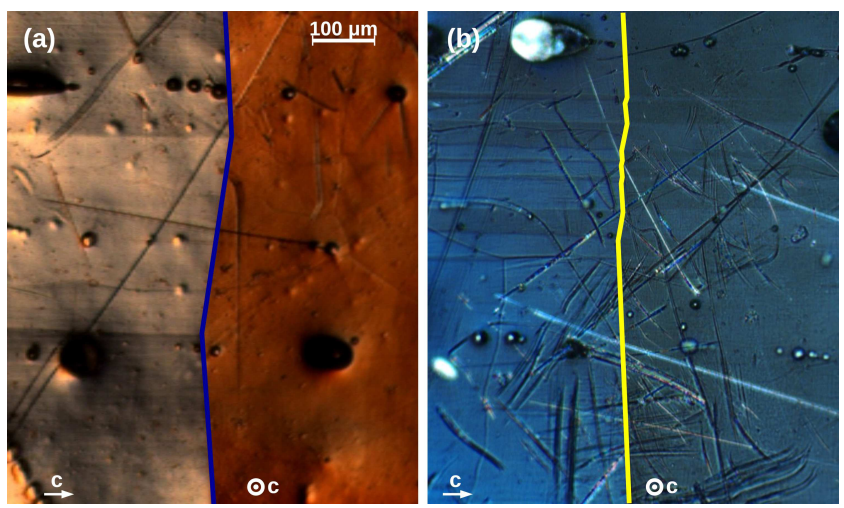

Fig. 3. Optical micrographs of type II twin boundary at the initial (a) and final (b) position. Trace of type II boundary, marked by a coloured vertical zigzag line, changes due to crossing monoclinic twin boundaries. The scale is same for all pictures.

As demonstrated in Fig. 3, the trace of type II twin boundary is different for the initial and final position. Two monoclinic twin boundaries cross the type II boundary at the initial position and cause two deflections of type II boundary. As the monoclinic twins are branched the amount of monoclinic boundaries increases and the type II boundary trace is more and more zigzagged to ac- before
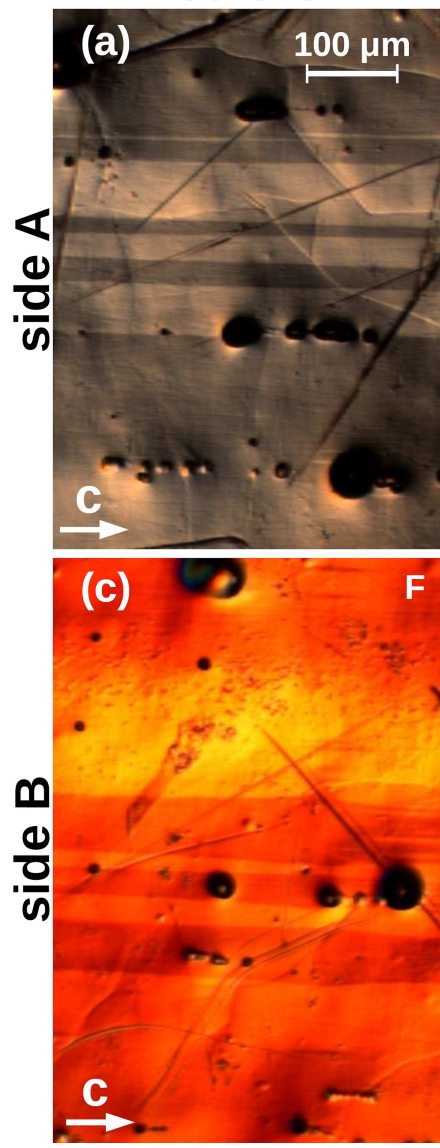

after
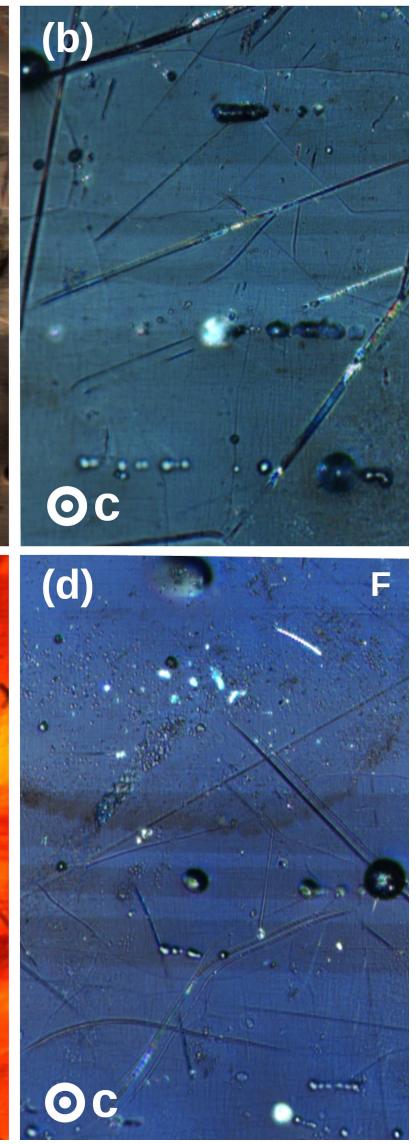

Fig. 4. Detail of monoclinic twinning before and after the twin boundary passage of both sample sides. Pictures (c) and (d) are vertically flipped (marked by F letter) to allow direct comparison with (a) and (b). The scale is the same for all pictures.

commodate branched monoclinic twinning. If even much more monoclinic boundaries would be present, the trace might look to be flat at optical microscope resolution as proposed by Heczko et al. [22].

The overview pictures at Fig. 2 indicate no or very minor changes of monoclinic twins after the passage of single twin boundary of type II. Therefore, we provide cut-out details of the original pictures to compare before and after the passage for side A and B (Fig. 4). Scratches and voids can serve as markers that the cut out sample areas are indeed identical. In this resolution, it seems that the monoclinic twins stayed about the same or just with very small changes. In contrast, magnetic domains change significantly after twin boundary passage from labyrinth to rake pattern [23].

Finally, we compare monoclinic twin microstructure on both sides. The side B pictures are flipped vertically to allow direct comparison with the side A pictures. The monoclinic twins do not differ much comparing side A and $\mathrm{B}$. This proves that the monoclinic twins propagate through the whole sample keeping coherency along (100) 
plane. It is in agreement and in some sense the extension of the model [13] and in contrast to TEM observation commonly showing curved modulation domains [16]. Observed curvature in TEM can be an artefact of the foil preparation.

The observation confirms that monoclinic twins stay unchanged after the passage of single twin boundary of type II at least on the resolution level of optical microscopy. The unchanged state indicates that compatibility between type II twin boundary and the proper monoclinic twinning boundary is very high in agreement with elastic compatibility calculation. Using higher resolution microscopic technique like scanning electron microscopy (SEM) may deliver more details. SEM could also bring informations about next level interactions between monoclinic twins and a/b laminate [21]. On the other hand, the TEM studies seem to be out of the question due to the high mobility of type II twin boundary, which make the preparation of such sample very difficult.

Although the observed changes are very small and often unobservable, the preliminary results indicate that many repeated passages of single twin boundary may produce changes in monoclinic twinning big enough to be detectable by optical microscopy. This suggests that repeated passage of twin boundary can change the monoclinic twin distribution. One may expect that this also change the distribution of $\mathrm{a} / \mathrm{b}$ twinning but these twins are not observable optically.

The opposite problem, how the presence of monoclinic twinning affect mobility of type II twin boundary, has not been yet studied. Detail measurement of twinning stress is needed in order to evaluate the effect of branching.

\section{Conclusion}

We showed that monoclinic twins in $10 \mathrm{M}$ martensite of $\mathrm{Ni}-\mathrm{Mn}-\mathrm{Ga}$ form macroscopic objects spreading across the whole sample. In contrast to previous studies in TEM, these are mostly straight and along (100) plane. The monoclinic twins configuration is not affected by the single passage of type II twin boundary, i.e after structural reorientation, significantly. Even few passages of twin boundary will not visibly modify monoclinic twinning. The question remains how the presence of monoclinic twins influence the twinning stress (mobility) of type II twin boundary. This study is the first step in the investigation of interaction dynamics between monoclinic twins and mobile a/c twin boundaries which may be important for any application. The results presented here for type II boundary should be extended using the same procedure for type I boundary.

\section{Acknowledgments}

This work was supported by Czech Science Foundation, grant No. 14-36566G and Grant Agency of the Czech Technical University in Prague, student grant No. SGS16/245/OHK4/3T/14.

\section{References}

[1] O. Heczko, N. Scheerbaum, O. Gutfleisch, in: Magnetic shape memory phenomena, Eds. J.P. Liu, E. Fullerton, O. Gutfleisch, D.J. Sellmyer, Springer US, New York 2009, p. 399.

[2] K. Bhattacharya, R.D. James, Science 307, 53 (2005).

[3] O. Heczko, A. Sozinov, K. Ullakko, IEEE Trans. Magn. 36, 3266 (2000).

[4] O. Heczko, Mater. Sci. Technol. 30, 1559 (2014).

[5] K. Ullakko, J. Mater. Eng. Perform. 5, 405 (1996).

[6] O. Söderberg, I. Aaltio, Y. Ge, O. Heczko, S.-P. Hannula, Mater. Sci. Eng. A 481, 80 (2008).

[7] G.B. Olson, Martensite, Ch. 1, AMS International, Materials Park 1992.

[8] J.W. Christian, The theory of transformations in metals and alloys, Ch. 1, Elsevier Science, Oxford 2002.

[9] J.A. Klostermann, J. Less-Common Met. 28, 75 (1972).

[10] J.S. Bowles, J.K. Mackenzie, Acta Met. 2, 129 (1954).

[11] L. Straka, O. Heczko, H. Seiner, N. Lanska, J. Drahokoupil, A. Soroka, S. Fähler, H. Hänninen, A. Sozinov, Acta Mater. 59, 7450 (2011).

[12] A. Sozinov, N. Lanska, A. Soroka, L. Straka, Appl. Phys. Lett. 99, 124103 (2011).

[13] H. Seiner, L. Straka, O. Heczko, J. Mech. Phys. Solids 64, 198 (2014).

[14] K. Bhattacharya, Microstructure of martensite: Why it forms and how it gives rise to the shape-memory effect, Ch. 5, Oxford University Press, New York 2003.

[15] V. Kopecký, O. Perevertov, L. Straka, M. Ševčík, O. Heczko, Acta Phys. Pol. A 128, 754 (2015).

[16] Y. Ge, H. Jiang, A. Sozinov, O. Söderberg, N. Lanska, J. Keränen, E. Kauppinen, V.K. Lindroos, S.-P. Hannula, Mat. Sci. Eng. A-Struct. 438, 961 (2006).

[17] O. Heczko, IEEE Trans. Magn. 50, 2505807 (2014).

[18] O. Heczko, V. Kopecký, L. Fekete, K. Jurek, J. Kopeček, L. Straka, H. Seiner, IEEE Trans. Magn. 51, (2015).

[19] R. Chulist, L. Straka, N. Lanska, A. Soroka, A. Sozinov, W. Skrotzki, Acta Mater. 61, 1913 (2013).

[20] Y. Ge, O. Heczko, O. Söderberg, S.-P. Hannula, Scr. Mater. 54, 2155 (2006).

[21] O. Heczko, L. Klimša, J. Kopeček, Scr. Mater. 131, 76 (2017)

[22] O. Heczko, J. Kopeček, L. Straka, H. Seiner, Mater. Res. Bull. 48, 5105 (2013).

[23] V. Kopecký, L. Fekete, O. Perevertov, O. Heczko, AIP Adv. 6, 56208 (2016) 